\title{
Controlling the Dominant Magnetic Relaxation Mechanisms for Magnetic Hyperthermia in bimagnetic core-shell nanoparticles
}

Fernando Fabris, ${ }^{a}$ Enio Lima Jr., ${ }^{a}$ Emilio De Biasi, ${ }^{a}$ Horacio E. Troiani, ${ }^{a}$ Marcelo Vásquez Mansilla, ${ }^{a}$ Teobaldo E. Torres, ${ }^{b, c}$ Rodrigo Fernández Pacheco, ${ }^{b, c}$ M. Ricardo Ibarra, ${ }^{b, c, d}$ Gerardo F. Goya, ${ }^{b, d}$ Roberto D. Zysler $^{a}$ and Elin L. Winkler ${ }^{a \dagger}$

We report a simple and effective way to control the heat generation of a magnetic colloid under alternate magnetic fields by changing the shell composition of bimagnetic core-shell $\mathrm{Fe}_{3} \mathrm{O}_{4} / \mathrm{Zn}_{x} \mathrm{Co}_{1-x} \mathrm{Fe}_{2} \mathrm{O}_{4}$ nanoparticles. The core-shell structure constitutes a magnetically-coupled biphase system, with an effective anisotropy that can be tuned by the substitution of $\mathrm{Co}^{2+}$ by $\mathrm{Zn}^{2+}$ ions in the shell. Magnetic hyperthermia experiments of nanoparticles dispersed in hexane and butter oil showed that the magnetic relaxation is dominated by Brown relaxation mechanism in samples with higher anisotropy (i.e., larger concentration of Co within the shell) yielding high specific power absorption values in low viscosity media as hexane. Increasing the $\mathrm{Zn}$ concentration of the shell, diminishes the magnetic anisotropy, which results in a change to a Néel relaxation that dominates the process when the nanoparticles are dispersed in a high-viscosity medium. We demonstrate that tuning the $\mathrm{Zn}$ contents at the shell of these exchange-coupled core/shell nanoparticles provides a way to control the magnetic anisotropy without loss of saturation magnetization. This ability is an essential prerequisite for most biomedical applications, where high viscosities and capturing mechanisms are present.

\section{Introduction}

The magnetic fluid hyperthermia (MFH) is a promising technique proposed as a coadjuvant oncological treatment in combination with radiotherapy and chemotherapy $^{1-3}$. This method is based on the heat generation by the magnetic losses of single-domain magnetic nanoparticles (MNPs) exposed to alternating magnetic fields. In this way, tumor cell death is promoted by the acute temperature increase in the cellular environment containing the MNPs. The heat-

\footnotetext{
${ }^{a}$ Instituto de Nanociencia y Nanotecnología, CNEA, CONICET, Centro Atómico Bariloche, Av. Bustillo 9500 (8400) S. C. Bariloche, Argentina.

${ }^{b}$ Instituto de Nanociencia de Aragón (INA),Universidad de Zaragoza, C/Mariano Esquillor S/N, 50018, Zaragoza, Spain.

${ }^{c}$ Laboratorio de Microscopias Avanzadas - LMA, Universidad de Zaragoza, C/Mariano Esquillor S/N, CP 50018, Zaragoza, Spain.

d Departamento de Física de la Materia Condensada, Facultad de Ciencias, C/ Pedro Cerbuna 12, 50009, Zaragoza, Spain.

† winkler@cab.cnea.gov.ar
}

ing efficiency is accounted by the Specific Power Absorption (SPA) or the Specific Loss Power (SLP) which quantifies the power absorption or dissipation per unit mass of magnetic material. The magnetic losses are originated from the phase shift between the nanoparticle's magnetic moment and the magnetic vector $\mathrm{H}$ of the applied alternating magnetic field, where the magnetic relaxation dynamics depend on the relaxation time of two different concurrent mechanisms ${ }^{4}$. One of them is the mechanical or Brownian relaxation, where the particles physically rotate with a characteristic time $\tau_{B}$, in presence of the alternating magnetic field. The second mechanism is the Néel relaxation, with a characteristic time $\tau_{N}$, which involves the inversion of the magnetic moment within the crystal lattice through its anisotropy energy barrier $K_{e f f} V$, where $K_{\text {eff }}$ is the effective anisotropy constant and $V$ 
is the volume of the particle ${ }^{5}$. It is known that the dominating relaxation process will be the one with the shorter relaxation time. In most cases, $\tau_{B}$ dominates for systems with large magnetic anisotropy energy and/or low viscosity, whereas $\tau_{N}$ governs in the opposite situation ${ }^{6}$.

Current experimental research is focused on the improvement of the heating power by optimizing the nanoparticles characteristic as the size ${ }^{7-9}$, morphology $^{10,11}$, magnetic anisotropy ${ }^{12}$, saturation magnetization $^{13}$ and also the surface functionalization ${ }^{14}$. Besides, different theoretical models have been proposed to calculate the SPA of magnetic nanoparticles in a different colloidal medium ${ }^{4-615-17}$ which allows to understand the main characteristics of the hyperthermia mechanisms and thus to predict the SPA under specific experimental conditions. However, although the experimental and theoretical advances in the field have been important, very few and specific cases have been approved for clinical use and still several problems must be resolved before its implementation can be effective and widespread approved.

One of the many hurdles to face is related to the variability of SPA results when experiments are performed in solution, cell culture or in vivo. Different factors affect the relaxation mechanisms, in particular the different viscosities of the media, the nanoparticles agglomeration inside different cell organelles or the nanoparticles fixation on cell membranes or extracellular tissue ${ }^{13,18-23}$, which inhibit the mechanical rotation. As a consequence, the magnetic relaxation is usually the dominant mechanism in magnetic hyperthermia in vivo or in conditions that emulate biological media. ${ }^{24-26}$ On the other hand, it was found that the cellular uptake is strongly influenced by the nanoparticle coating material ${ }^{27}$. In this complex scene it is crucial to be capable to perform a selective optimization of individual parameters that control the magnetic relaxation mechanisms to improve the SPA for each particular experimental condition. One way to reach this goal is through the design and fabrication of bimagnetic core/shell nanoparticles which allows the systematic modification of the magnetic anisotropy, preserving the size, morphology and high values of magnetization ${ }^{28-30}$. Previous stud- ies have shown that bimagnetic nanostructures composed by hard and soft magnetic materials rigidly coupled by exchange interaction, have an effective magnetic anisotropy that results from the average of the magnetic anisotropies of each phase ${ }^{31-36}$. This result provides an additional tool to perform a fine control of the effective magnetic anisotropy of the nanoparticles, by changing the characteristic of one of the phases. In this way the magnetic relaxation time can be modified respect to the mechanical relaxation and the dominant Brown or Néel heating mechanism could be tuned.

In this work, with the aim to control the mechanism of the heat generation in $\mathrm{MFH}$, we have fabricated core/shell nanoparticles composed by $\mathrm{Fe}_{3} \mathrm{O}_{4} / \mathrm{Zn}_{x} \mathrm{Co}_{1-x} \mathrm{Fe}_{2} \mathrm{O}_{4}$, with $x$ varying from 0 to 1 . As the $\mathrm{Zn}$ concentration increases, the shell magnetic anisotropy is reduced; as a consequence, the effective magnetic anisotropy of the system can be controlled by changing the shell stoichiometry. The magnetic hyperthermia was evaluated in two medium of low and high viscosity: hexane $(0.3 \mathrm{mPa} . \mathrm{s} \text { at } 295 \mathrm{~K})^{37}$ and butter oil (476 mPa.s at $295 \mathrm{~K})^{38}$, respectively. The results clearly show a change in the dominant relaxation mechanisms of magnetization in MFH experiments, from Brown to Néel relaxation, in agreement with the diminution of the effective magnetic anisotropy when the $\mathrm{Zn}$ concentration at the shell increases. These results show the enormous potential of core/shell systems to develop nanoparticles with specific characteristics for different environmental conditions to optimize SPA values.

\section{Experimental}

Nanoparticles were prepared by the seed-mediated thermal decomposition of organo-metallic precursors at high temperature as described elsewhere ${ }^{39,40}$. Briefly, the $\mathrm{Fe}_{3} \mathrm{O}_{4}$ core is synthesized from $12 \mathrm{mMol}$ of $\mathrm{Fe}(\mathrm{III})$ acetylacetonate in presence of $24 \mathrm{mMol}$ of 1,2octanediol, $8 \mathrm{mMol}$ of Oleic acid, $40 \mathrm{mMol}$ of Oleylamine and $210 \mathrm{~mL}$ of Benzyl ether as solvent. The solution was heated up to $300{ }^{\circ} \mathrm{C}$ (reflux condition) during $1 \mathrm{~h}$ under a $\mathrm{N}_{2}$ flow $(0.1 \mathrm{~mL} / \mathrm{min}$.) and intense mechanical stirring. After the cooling process, five fractions of $10 \mathrm{~mL}$ each were separated to overgrowth 
the shell, and the remaining solution $\mathrm{Fe}_{3} \mathrm{O}_{4}$ nanoparticles was kept as a control of the as-synthesized core. Core-shell nanoparticles were prepared in a similar reaction, but in presence of the core that acts as seeds for the growing of the $\mathrm{Zn}_{x} \mathrm{Co}_{1-x} \mathrm{Fe}_{2} \mathrm{O}_{4}$ shell. Five coreshell samples were prepared with $x=1.00,0.75,0.50$, 0.25 and 0.00 . For this, the $8.2 \mathrm{~mL}$ solution containing the core were added to $(1-x) \times 0.6 \mathrm{mMol}$ of $\mathrm{Co}$ (II) acetylacetonate, $x \times 0.6 \mathrm{mMol}$ of $\mathrm{Zn}$ (II) acetylacetonate, $1.2 \mathrm{mMol}$ of Fe(III) acetylacetonate, $3 \mathrm{mMol}$ of 1,2 octanediol, $3 \mathrm{mMol}$ of oleic acid, $3 \mathrm{mMol}$ of oleylamine and $210 \mathrm{mMol}$ of benzyl ether. After the synthesis, the nanoparticles were precipitated by adding 8 times in volume of a solution containing ethanol and acetone $(4: 1)$ followed by centrifugation (14000 rpm during 30 minutes). Finally, the samples in powder form were dispersed in hexane.

Dynamic Light Scattering (DLS) measurements of nanoparticles diluted in hexane were performed in a ZetaSizer from Malvern Instruments with a measurements angle of $90^{\circ}$. In a total, 3 measurements were performed, each one composed by 20 runs.

Transmission Electron Microscopy (TEM) images were taken in a thermo-ionic LaB6 Tecnai T20 microscope operating at an accelerating voltage of 200 $\mathrm{kV}$. Scanning Transmission Electron Microscopy - High Angle Annular Dark Field (STEM-HAADF) images were obtained in a Cs-probe-corrected Titan (ThermoFisher Scientific, formerly FEI) at a working voltage of $300 \mathrm{KV}$, coupled with a HAADF detector (Fischione). In order to obtain the chemical composition of the nanoparticles, Electron Energy Loss spectra (EELS) were obtained with a Tridiem Energy Filter (Gatan) as spectrometer. The sample was scanned with a fine probe and chemical profiles were obtained for each of the elements by extracting the corresponding absorption energy from each EEL spectrum. For this, the nanoparticles suspension were diluted and drops were disposed on a copper grid with an ultrathin hollow carbon film and subjected to plasma cleaning during 10 seconds in order to reduce the presence of organic components remaining from the synthesis.

Magnetization curves as function of temperature (M(T)) with applied field of 20 Oe were measured in a SQUID magnetometer (MPMS 5000 from Quantum Design), by using the Zero-field-cooling (ZFC) and Field-cooling (FC) protocols. For these measurements, the nanoparticles were dispersed with a concentration of $0.5 \%$ wt. in an epoxy resin.

Magnetic fluid hyperthermia experiments were performed in DM100 applicator from nB - NanoScaleBiomagnetics, with an ac applied field of 200 Oe and different frequencies: $228 \mathrm{kHz}, 350 \mathrm{kHz}, 570 \mathrm{kHz}$ and $817 \mathrm{kHz}$. The measurements start at room temperature (about $295-297 \mathrm{~K}$ ) and the temperature increment was measured with an optical thermometer. Two different media were used: the first one consists in $0.5 \%$ wt. of nanoparticles dispersed in hexane $(\eta(295 K)=0.3 \mathrm{mPa} . \mathrm{s})^{37}$, while the second one is the $0.5 \%$ wt nanoparticles dispersion in butter oil $(\eta(295 K)=477 \text { mPa.s })^{38}$. This second solution was prepared by the mixture of the same volume of butter oil and the hexane solution followed by the total evaporation of the hexane at $323 \mathrm{~K}$.

\section{Results and discussion}

Figure 1(a) shows the TEM image of the $\mathrm{Fe}_{3} \mathrm{O}_{4}$ core samples where uniform shape is observed. The corresponding size histograms fitted with a lognormal distribution (right panel), yielding a mean core diameter of $\left\langle d_{\text {core }}\right\rangle=7 \mathrm{~nm}$. The TEM images of the core/shell nanoparticles are shown in Figure 1(c-f) and Figure S1 of the Supplementary Information. From the size histograms, shown in Figure 1(d) and Figure S2 (see Supplementary Information), the mean diameters for all the systems were found to be within the 9-10 nm size values (see Table 1). Although the lattice parameters of both $\mathrm{Fe}_{3} \mathrm{O}_{4}$ and $\mathrm{Zn}_{x} \mathrm{Co}_{1-x} \mathrm{Fe}_{2} \mathrm{O}_{4}$ spinel phases are similar and the phases cannot be resolved from the electron or X-ray diffraction pattern, the shell overgrown with different crystalline orientation relative to the core. Therefore, it is possible to differentiate the shell from the core as observed from the HRTEM images for $x=0.00$ and 1.00 in Figure 1 and Figure S3, respectively. The core/shell structure is also unveiled in the dark field image, reconstructed from a fraction of the (133) spinel diffraction ring, where the bright image correspond to the grains with the selected crystallographic orientation. From the TEM image and the 


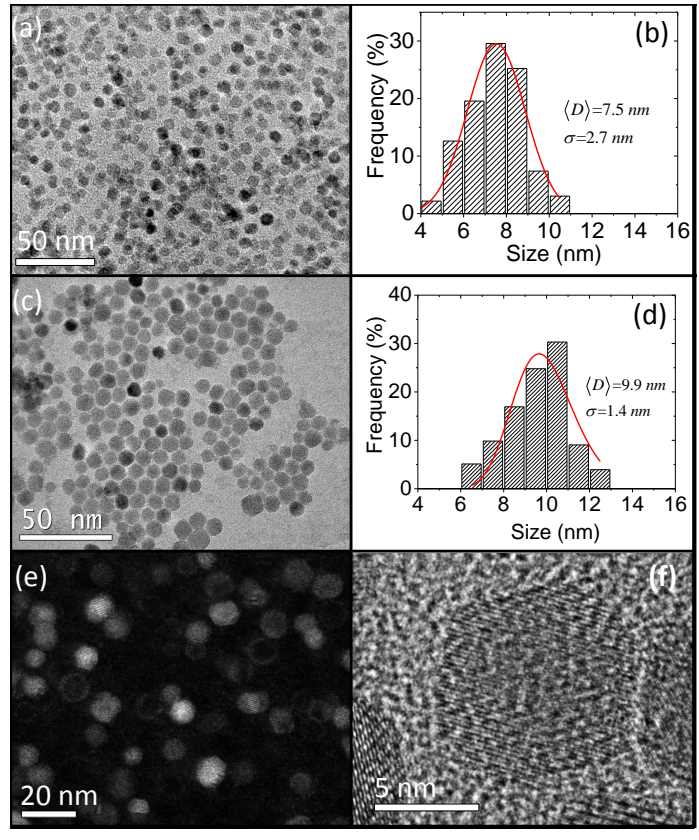

Fig. 1 Representative TEM images with the corresponding size histograms of the $\mathrm{Fe}_{3} \mathrm{O}_{4}(\mathrm{a}-\mathrm{b})$ and $\mathrm{Fe}_{3} \mathrm{O}_{4} / \mathrm{CoFe}_{2} \mathrm{O}_{4}$ (c-d) nanoparticles. The histograms where fitted with a lognormal distribution (red line). Dark field image (e) of and HRTEM image (f) corresponding to the core/shell sample with $x=0.00$, where the core shell structure is evidenced.

Table 1 Morphologic and magnetic parameters for the core and the $\mathrm{Fe}_{3} \mathrm{O}_{4} / \mathrm{Zn}_{x} \mathrm{Co}_{1-x} \mathrm{Fe}_{2} \mathrm{O}_{4}$ core/shell the samples.

\begin{tabular}{lcccccc}
\hline Sample & $\langle d\rangle$ & $\sigma$ & $K_{\text {eff }}$ & $\left\langle T_{B}\right\rangle$ & $\langle\mu\rangle$ & $d_{\text {hyd }}^{\text {exp }}$ \\
& $\mathrm{nm}$ & $\mathrm{nm}$ & $10^{5} \frac{\mathrm{erg}}{\mathrm{cm}^{3}}$ & $\mathrm{~K}$ & $\frac{\text { emu }}{\mathrm{g}}$ & $\mathrm{nm}$ \\
\hline Core & 7.5 & 2.5 & - & - & - & - \\
0.00 & 9.9 & 1.4 & 6.6 & 152 & 107 & 16 \\
0.25 & 10.0 & 2.0 & 6.3 & 140 & 93 & 10 \\
0.50 & 9.4 & 1.4 & 6.0 & 134 & 105 & 10 \\
0.75 & 9.4 & 1.3 & 3.2 & 68 & 95 & 18 \\
1.00 & 9.7 & 1.4 & 0.7 & 21 & 96 & 22 \\
\hline
\end{tabular}

difference between the mean diameter of the core and core/shell particles the shell thickness for each sample was determined, which vary in the $1.3-1.6 \mathrm{~nm}$ range along the series.

The compositional analysis was performed by electron energy loss spectroscopy (EELS). EELS mapping of samples $x=0.00,0.50$ and 1.00 are presented in Figure 2. For sample $\left(\mathrm{Fe}_{3} \mathrm{O}_{4} / \mathrm{CoFe}_{2} \mathrm{O}_{4}\right.$ ) (upper panel), a Co-Fe oxide shell is evident through the presence of the absorption energy edges for $\mathrm{Fe}(708 \mathrm{eV})$ and Co $(779 \mathrm{eV})$. The Co energy peak is also observed at the core of the particles but with lower intensity when compared to the shell. It is noteworthy that the EELS signal at the middle of the particle arises from both the core and the shell which makes difficult to isolate the core information, however the different intensity of the Co peak at the core and shell agrees with the nominal composition. For the sample $x=1.00$ $\left(\mathrm{Fe}_{3} \mathrm{O}_{4} / \mathrm{ZnFe}_{2} \mathrm{O}_{4}\right)$, showed in the bottom panel, besides the absorption energy edges for $\mathrm{Fe}$, a large signal from the Zn L edge (1020 eV) was detected at both the core and the shell particle. Instead, for intermediate concentration although the EELS point spectrum clearly confirms the presence of magnetite, the $\mathrm{Co}$ and $\mathrm{Zn}$ peaks are hard to be noted. For example for $x=0.50\left(\mathrm{Fe}_{3} \mathrm{O}_{4} / \mathrm{Zn}_{0.5} \mathrm{Co}_{0.5} \mathrm{Fe}_{2} \mathrm{O}_{4}\right)$, the $\mathrm{Zn}$ signal at the shell is really weak; and only some small hub at 1020 $\mathrm{eV}$ can be detected, while the spectrum clearly indicates the presence of Fe. Therefore, although the technique does not allow us to make a local compositional analysis, qualitatively supports the multi-metallic nature of the shell.

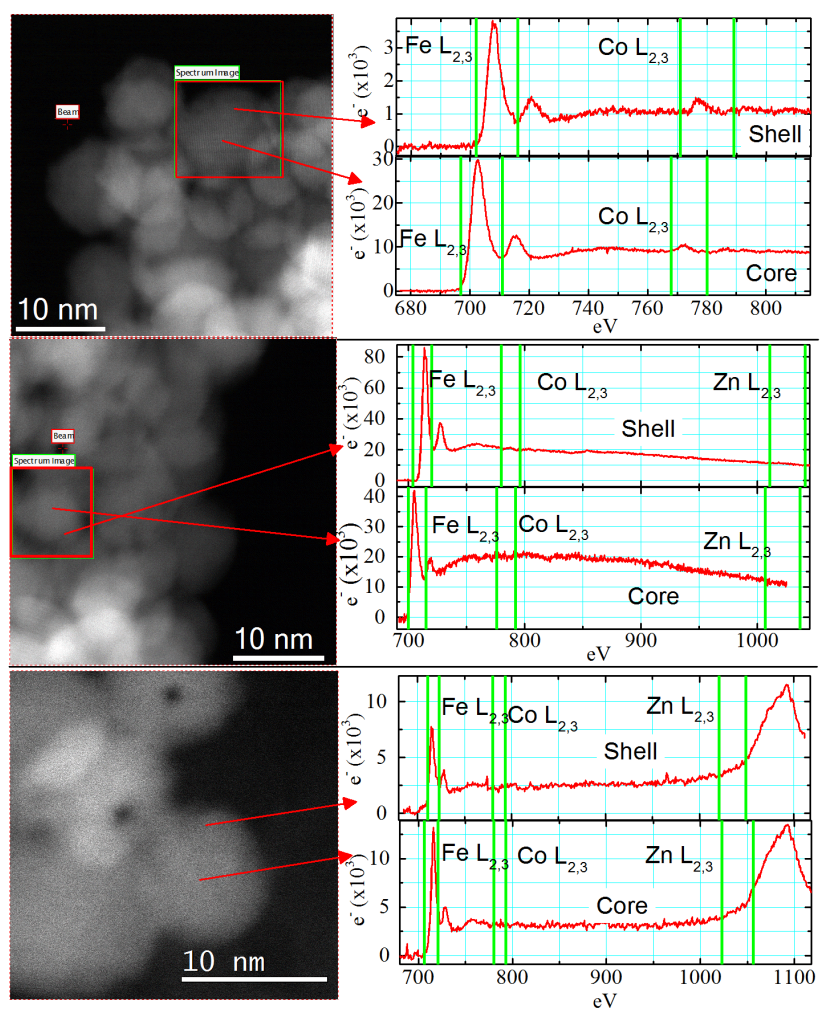

Fig. 2 EEL spectra for all samples obtained from the spectrum image (EELS mapping) for the core and for the shell regions of samples $x=0.00$ (upper panel), $x=0.50$ (middle panel) and $x=1.00$ (bottom panel). 


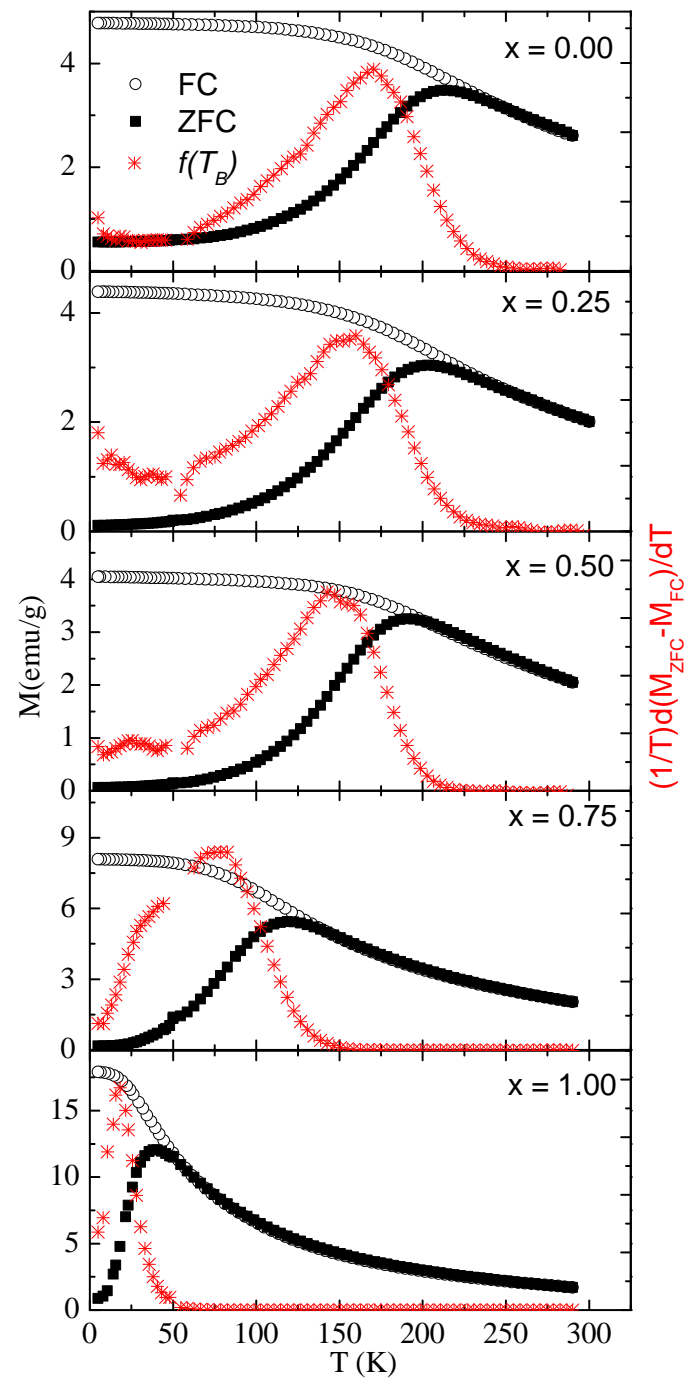

Fig. $3 \mathrm{M}(\mathrm{T})$ curves measured at FC and ZFC protocols with $\mathrm{H}=20 \mathrm{Oe}$. The distributions of blocked temperature $f\left(T_{B}\right)$ for each sample calculated as $(1 / T) d\left(M_{Z F C}-\right.$ $\left.M_{F C}\right) / d T$, are also shown.

Figure 3 shows the temperature dependence of the ZFC and FC magnetization of the systems. All samples present an irreversible behavior at low temperature and a maximum in the ZFC curves that shifts to lower temperature when the concentration of $\mathrm{Zn}$ increases. These features are characteristics of a change from superparamagnetic to blocked regime, where the blocking temperature $\left(T_{B}\right)$ systematically decreases with the $\mathrm{Zn}$ concentration. The distribution of blocking temperature of the nanoparticle system, $f\left(T_{B}\right)$, can be calculated from the magnetization curves by $(1 / T) d\left(M_{Z F C}-M_{F C}\right) / d T$, as shown in Figure 3 . It is noteworthy that the $f\left(T_{B}\right)$ expression is valid for non-interacting nanoparticles and for coher-

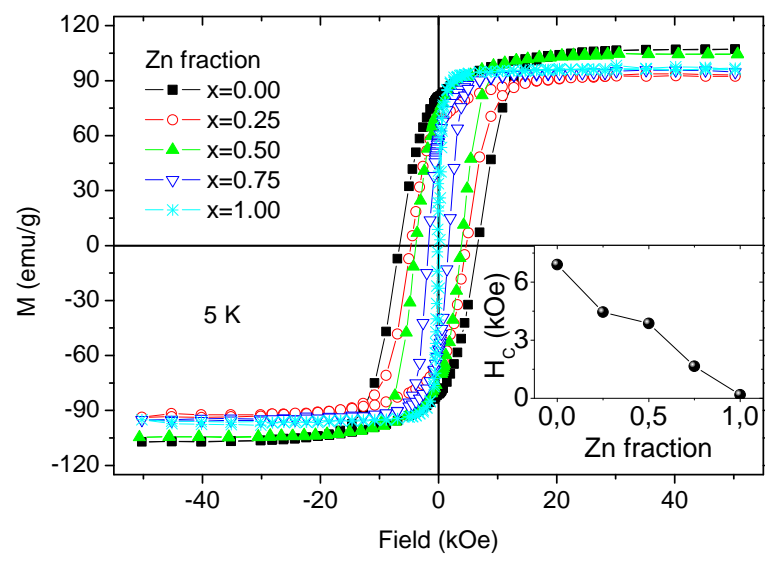

Fig. 4 Hysteresis loops of all samples $(M(H))$ measured at $5 \mathrm{~K}$. In the inset the $H_{C}$ as function of the Zinc fraction in the shell is shown.

ent magnetic inversion of the magnetic moment of the particle. In the present system this approximation implies a coherent relaxation of both, core and shell, magnetic phases rigidly coupled by exchange interaction. Since the blocking temperature of a single particle is given by its energy barrier, then $f\left(T_{B}\right)$ is the result of a combination of the size and the anisotropy distributions. Generally, as the size distribution is broader than the anisotropy distribution, the $f\left(T_{B}\right)$ can be directly related to the size distribution by using a mean value of $K_{e f f}$. Therefore, the size distribution can be reconstructed from the $M_{F C}$ and $M_{Z F C}$ curves. For comparison Figure S2 of Supplementary Information shown the diameter distributions obtained in this way superimposed to the corresponding TEM histograms for each sample. The good agreement between the particle diameter distribution obtained from $f\left(T_{B}\right)$ and the size histograms obtained from TEM images support the coherent reversion of the nanoparticles magnetic moment. In addition, this procedure gave us a more accurate value of the anisotropy constant $K_{\text {eff }}$, (see Table 1 ) which decreases with the shell composition from $6.6 \times 10^{5}$ $\mathrm{erg} / \mathrm{cm}^{3}$ for $x=0.00$ to $0.7 \times 10^{5} \mathrm{erg} / \mathrm{cm}^{3}$ for $x=1.00$. This result is consistent with the decreases of the shell magnetic anisotropy from $4 \times 10^{6} \mathrm{erg} / \mathrm{cm}^{3}$ to $1 \times 10^{4}$ $\mathrm{erg} / \mathrm{cm}^{3}$ for $\mathrm{CoFe}_{2} \mathrm{O}_{4}$ and $\mathrm{ZnFe}_{2} \mathrm{O}_{4}$, respectively.

The magnetization loops measured at $5 \mathrm{~K}$, are presented in Figure 4, where a single reversion curve for all the nanoparticles systems can be observed. This 
behavior is also in agreement with the coherent rotation of the coupled magnetizations of both phases. The inset shows the coercive field $\left(H_{C}\right)$ as a function of $x$ measured at $5 \mathrm{~K}$, where a systematic decrease with the $\mathrm{Zn}$ concentration is observed, from 6800 Oe for $x=0.00$, to 190 Oe for $x=1.00$. This evolution can be accounted by the average coercivity expected for a powder of rigid coupled hard/soft magnetic nanoparticles $28,31,32,34,36$ :

$$
H_{C}=\frac{K_{C} f_{C}+K_{S h} f_{S h}}{M_{C} f_{C}+M_{S h} f_{S h}}
$$

where $M$ is the saturation magnetization, $f$ is the volume fraction of the core (C) and shell (Sh), respectively. From the saturation magnetization and the magnetic anisotropy of each component as well as the volumetric fraction estimated from TEM analysis, the coercive field from Equation 1 results approximately $10 \mathrm{kOe}$ for the $\mathrm{Fe}_{3} \mathrm{O}_{4} / \mathrm{CoFe}_{2} \mathrm{O}_{4}$ sample and systematically decreases to 200 Oe for the $\mathrm{Fe}_{3} \mathrm{O}_{4} / \mathrm{ZnFe}_{2} \mathrm{O}_{4}$ system. Although Equation 1 qualitatively explains the experimental results, overestimate the coercivity field, because it is deduced from an oversimplified model where the temperature effect is not considered and assumes perfect and flat interfaces where the phases are rigidly coupled with easy axis collinear. ${ }^{41}$ It is evident from Figure 4 that the saturation magnetization of all the samples remains almost constant as the shell stoichiometry changes. Although in bulk material the saturation magnetization of $\mathrm{Zn}_{x} \mathrm{Co}_{1-x} \mathrm{Fe}_{2} \mathrm{O}_{4}$ decreases with the $\mathrm{Zn}$ concentration, in nanostructures the size effects, such as disoredered cation distribution ${ }^{42-44}$ and oxygen vacancies ${ }^{45}$, usually yields an increase of magnetization; and these mechanisms could explain why the saturation magnetization of core/shell nanoparticles remains almost unchanged with $x$.

Figure 5 shows the results of the magnetic fluid hyperthermia experiment of the nanoparticles dispersed in hexane ( $\eta=0.3 \mathrm{mPa} . \mathrm{s})$ measured with a field amplitude $\left(H_{0}\right)$ of 200 Oe and four different frequencies $(228,350,570$ and $818 \mathrm{kHz})$. The figure gives the variation of the temperature $\Delta T$ with time, with respect to the initial room temperature $\approx 295 \mathrm{~K}$. From the slope of the initial part of the $\Delta T(t)$ curve, we have estimated the Specific Power Absorption by: $S P A=$
$\left(C_{l i q} \cdot m_{l i q} / m_{N P S}\right) d T / d t$, where $C_{l i q}$ and $m_{l i q}$ are the specific heat and the mass of the liquid, respectively, and $m_{N P s}$ is the mass of the nanoparticles. In our experiments, we use the relation $m_{l i q} / m_{N P s}=1 / 0.005$ for all samples, as mentioned in the Experimental Procedure section.
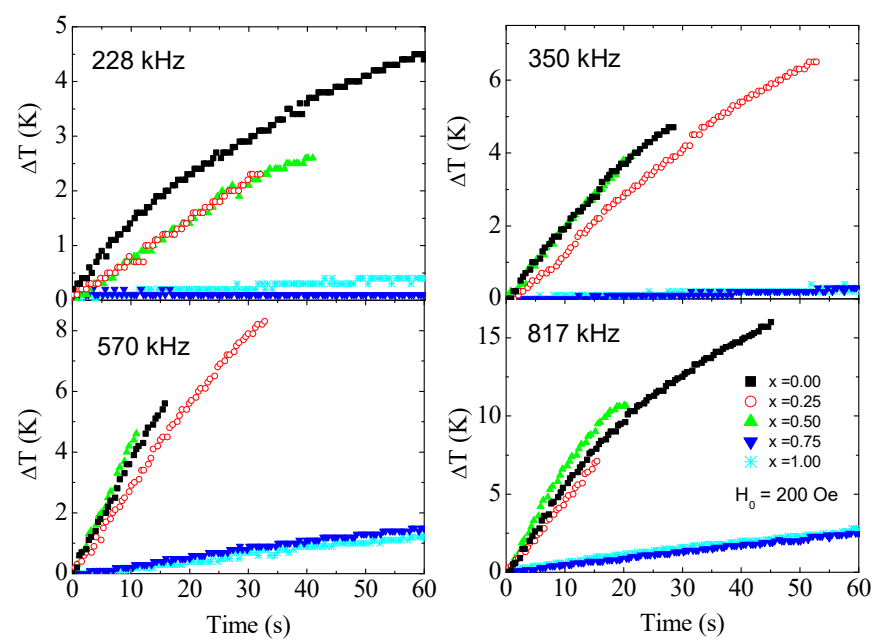

Fig. 5 Magnetic fluid hyperthermia experiments for the nanoparticles systems dispersed in Hexane $(\eta=0.3$ $\mathrm{mPa} . \mathrm{s})$ measured with four different frequencies $(228,350$, 570 and $818 \mathrm{kHz}$ ) and field amplitude $H_{0}$ of $200 \mathrm{Oe}$.

The SPA values obtained as a function of the $\mathrm{Zn}$ concentration are given in Figure 6(a). It is remarkable that, for all the frequencies explored, the evolution of the SPA with the $\mathrm{Zn}$ concentration shows a sharp drop within the $0.5<x<0.75$ concentration range, more visible at the highest frequency where the SPA drops from $\approx 200 \mathrm{~W} / \mathrm{g}(x=0.5)$ to $10 \mathrm{~W} / \mathrm{g}(x=0.75)$. This result reflects a change in the mechanism of magnetic relaxation between the samples with Co rich shell $(x \leq 0.5)$ and those with Zn rich shell $(x \geq 0.75)$.

As all the systems present comparable nanoparticles size and organic coating, a similar Brown relaxation time, defined as $\tau_{B}=3 \eta V_{\text {hyd }} / k_{B} T$ is expected; where $\eta$ is the viscosity of the medium, $k_{B} T$ is the thermal energy and $V_{h y d}$ is the hydrodynamic volume of the particle (see Figure S4 of the Supplementary Information). On the other hand, when the $\mathrm{Zn}$ concentration at the shell increases, the effective magnetic anisotropy decreases, and consequently the Néel relaxation time $\tau_{N}=\tau_{0} \exp \left(K_{e f f} V / k_{B} T\right)$ is shorter, where $\tau_{0}$ is the characteristic magnetic relaxation time of 
the material. Therefore, as $\tau_{N}$ diminishes the system would display a transition from the Brownian relaxation to Néel relaxation mechanism when $\tau_{N}<\tau_{B}$. As a consequence the sharp drop of the SPA observed at $0.5<x<0.75$ (Figure 6) is consistent with a change of relaxation process from mechanical, for $x \leq 0.5$, to magnetic relaxation dynamic for $x \geq 0.75$.
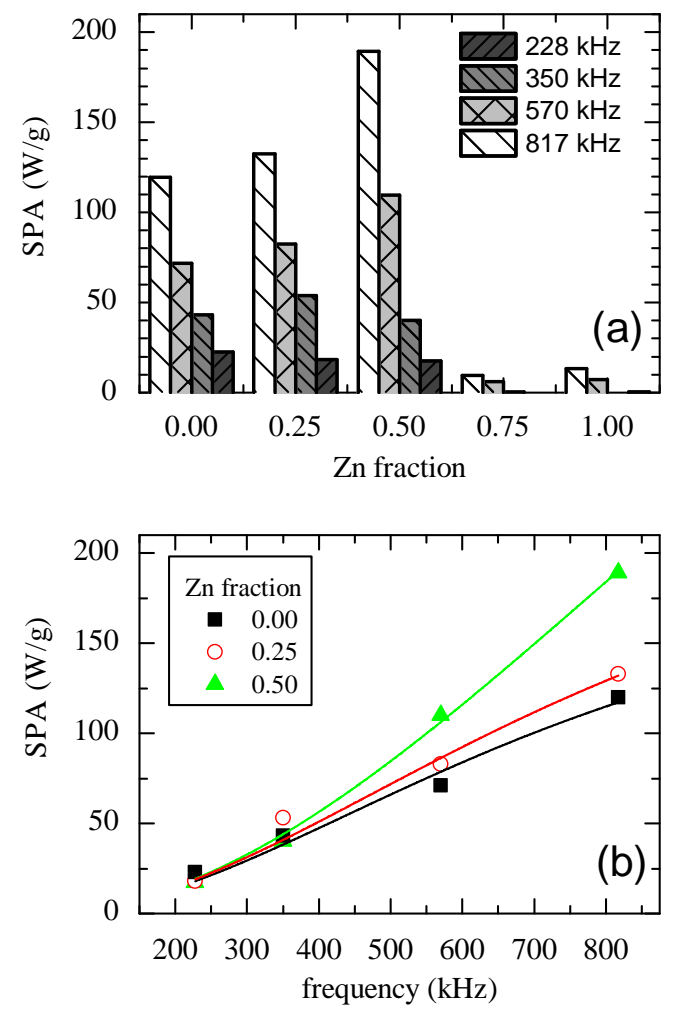

Fig. 6 (a) SPA values as function of the $\mathrm{Zn}$ concentration $(x)$ at the shell, measures at different frequencies. (b) SPA values as function of the frequency for samples with $x \leq 0.5$. The solid line represents the fit with eq. 2 .

In order to confirm this picture, we performed the hyperthermia experiments using a medium with larger viscosity as the butter oil ( $\eta \approx 476 \mathrm{mPa} . \mathrm{s}$ ). The increase of more than three orders of magnitude in the viscosity would produce a proportional increase in the Brown relaxation time, and therefore a drop of SPA is expected if this is the dominant heating mechanism. Figure 7 gives the comparison between the SPA values obtained for hyperthermia experiments performed for nanoparticles dispersed in hexane and in butter oil. As observed, the SPA values are reduced for $x \leq 0.5$ samples from values between $120-180 \mathrm{~W} / \mathrm{g}$ to $1-4 \mathrm{~W} / \mathrm{g}$ for the MNPs dispersed in hexane and butter oil, respectively. This result confirms that for the $x \leq 0.5$ samples the dominant mechanism is the Brown relaxation. On the contrary, the samples with $x \geq 0.75$ present SPA values in butter oil as small as the observed for hexane, reinforcing the conclusion that these samples have different relaxation mechanisms, probably strictly magnetic, which are not optimized for the frequency range used in the experiments.

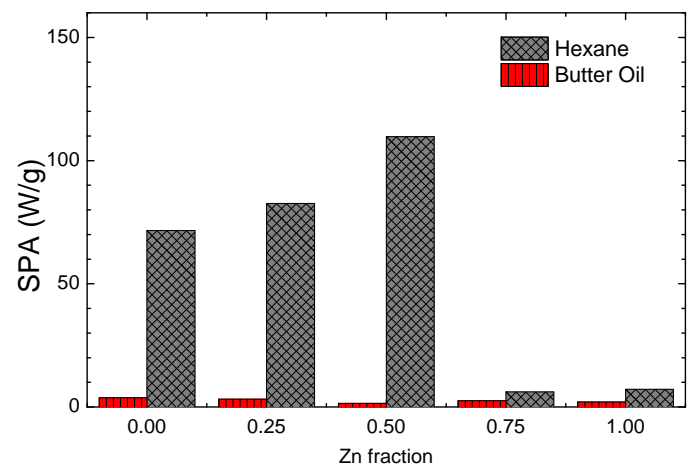

Fig. 7 SPA values obtained from the magnetic fluid hyperthermia experiments for nanoparticles dispersed in hexane and in butter oil, measured from $T_{0}=294 \mathrm{~K}$ with $H_{0}=200$ Oe and $f=570 \mathrm{kHz}$.

A complete map of the mechanisms involved in the magnetic moment relaxation of the particles can be obtained using the relaxation time diagram presented by Lima et $a l^{6}$. This diagram, shown in 8 , describes the relation between $\tau_{f} / \tau_{B}$ and $\tau_{f} / \tau_{N}$, where $\tau_{f}=1 /(2 \pi f)$ is the characteristic time of the measurements. The diagonal dashed lines of the figure divide the diagram in three regions: the upper-left corresponds to the systems in which the Néel mechanism dominates, in the low-right regions the Brown mechanism dominates; and in the central region both process are important. Considering the morphological and magnetic characteristic of the core/shell nanoparticles, the viscosity of the media and the measurement frequency, we can calculate the $\tau_{f} / \tau_{B}$ and $\tau_{f} / \tau_{N}$ relations for our particular system and placed these data in Figure 8. The open and solid dots correspond to the nanoparticles dispersed in hexane and butter oil, respectively, and the arrows indicate the increasing sense of the frequency (from 228 to $817 \mathrm{kHz}$ ). This figure evidences that for nanoparticles with $x=0.00$, 0.25 and 0.50 the Brown mechanism dominates the relaxation process when they are dispersed in hex- 
ane. Instead, for nanoparticles with $x=0.75$ both mechanisms are relevant, and for $x=1.00$ the Néel mechanism dominates. This scheme clearly shows as the control of the effective anisotropy changes the relaxation channel. Instead, when the nanoparticles are dispersed into butter oil, the mechanical rotation freeze, the $\tau_{f} / \tau_{B}$ parameter decreases and, as a consequence, the dominant relaxation is the magnetic one, as schematized in Figure 8 where the solid dot are located in the Néel relaxation zone for all composition. With the aim to evaluate the heating mechanism of this system in physiological condition, we have dispersed the core/shell nanoparticles in DMEM solution, a cell culture medium commonly used. To disperse the nanoparticles in the medium, the hydrophobic character of the nanoparticles was changed to hydrophilic by coating the nanoparticles with polyethylene glycol (PEG) as reported in Ref. ${ }^{46}$. As shown in Figure S5 of Supplementary Information, such procedure leads to a greater hydrodynamic radius due the nanoparticles PEG coating. This difference makes difficult a straightforward comparison of the heating process between the hydrophilic and hydrophobic nanoparticles. Anyway, from the experimental parameters the relaxation times can be evaluated,as shown in the Figure S6 of the Supplementary Information, which indicated that the dominant mechanism of the nanoparticles dispersed in DMEM is the Néel relaxation.

The analysis of the dominant relaxation mechanisms in terms of characteristic times, presented above, has been further completed by using Rosensweig's model to calculate the SPA value as a function of the rheological, morphologic and magnetic properties of the system. This approach is based on the assumption that the particles have a coherent inversion of the magnetic moment and the magnetization response linearly with the alternating magnetic field. These approximations are accounted as: $\xi=M_{S} V H_{0} / k_{B} T$ and $h=H_{0} /\left(H_{k}(f, T)\right) \ll 1^{5}$. In this model the specific power absorption results:

$$
S P A=A \frac{2 \pi f \tau}{1+(2 \pi f \tau)^{2}}
$$

where $A=\pi \chi_{0} f H_{0}^{2}$, and $\chi_{0}$ is the initial susceptibility.

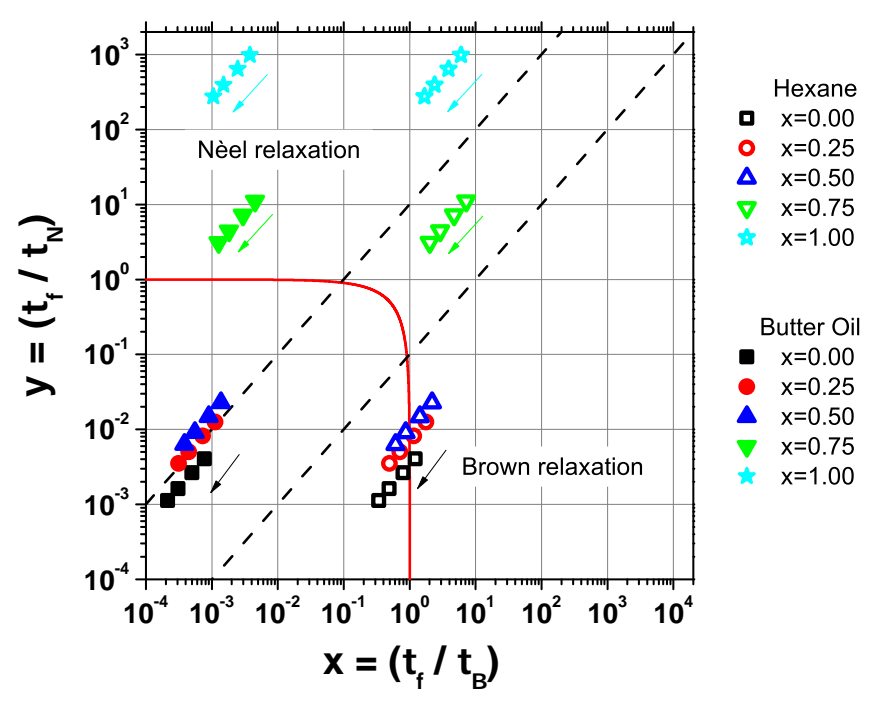

Fig. 8 Diagram of mechanisms of magnetic relaxation. Empty dots and full dots correspond to the samples diluted in hexane and butter oil, respectively. The arrows indicate the direction of increasing frequency. All data were obtained from the magnetic and morphological characterizations. $\tau_{f}=1 /(2 \pi f)$, where $f$ is the frequency of the experimental applied field. The red line corresponds to the optimum condition $2 \pi f \tau=1$ required to reach the maximum SPA intensity.

The above equation predicts the optimum relation between the characteristic time of the experiment and the relaxation time of the system to reach the maximum Specific Power Absorption of the system when $2 \pi f \tau=1$. This condition is represented by a red line in Figure 8, and provides, by a simple inspection a tool to adjust the measurement frequency, or the physical parameters of the system, to optimize the SPA value. In order to analyze our results from this picture we should verify that the mentioned hypotheses are fulfilled for the present core/shell nanoparticles. The $h$ and $\xi$ parameters were calculated for all the samples and presented in Table S1 of the Supplementary Information. It is observed that $h$ is smaller enough than 1 , but $\xi$ is in the $1-1.5$ range. However, this last hypothesis guarantees the use of the Curie susceptibility as the equilibrium limit of the magnetization which, if not fulfilled, can be corrected. Therefore, the Rosensweig's model can be applied to the present core/shell nanoparticles to obtain a qualitative and comparative description of the system. According to this picture, when the data of the nanoparticles over- 
lap the red line in Figure 8, the system should present the larger SPA value, as the samples $x=0,0.25$ and 0.50 dispersed in hexane. On the contrary, the samples $x=0.75$ and $x=1.00$ are farther away from the ideal heating condition, therefore their SPA should be smaller. This analysis is consistent with the experimental results shown in Figures 5 and 6, where an abrupt SPA decrease is observed for $x>0.5$. Moreover, assuming that the Brown relaxation is the dominant mechanism for $x \leq 0.5$, the frequency dependence of the SPA can be well fitted with Eq. 2, as shown in Figure 6(b). The fitting were done considering $V_{\text {hyd }}$ and $A$ as adjusting parameters. From $V_{\text {hyd }}$ we have obtained the hydrodynamic diameter of 19, 24 and 24 nm for the samples $x=0.00,0.25$ and 0.50 , respectively which are in agreement with the hydrodynamic diameter measured by DLS in a Hexane solution $\left(d_{\text {hyd }}^{\text {exp }}\right)$ reported in Table 1.

Finally when the particles are dispersed in butter oil its relaxation behavior, represented by the solids dots in Figure 8, shifts to the Néel relaxation zone and away from the optimal heating condition. This observation is in agreement with the experimental results presented in Figure 7 where a strongly diminution of its heating rate is observed when the particles are diluted in butter oil, compared to its optimum value reached when they are dispersed in hexane. Similar behaviour is presented by the hydrophilic nanoparticles dispersed in DMEM, where the magnetic anisotropy is away from the optimun value and as a consequence this system present low SPA. These results show that, by modulating the magnetic anisotropy of the nanoparticles, we can tune not only the Brown or Néel relaxation mechanism but also adjust the condition to obtain the maximum heating.

\section{Conclusion}

In this work we showed that the magnetic relaxation mechanism can be tuned by a systematic change of the effective magnetic anisotropy in a core/shell nanoparticles system. By changing the shell composition in a strongly exchange-coupled $\mathrm{Fe}_{3} \mathrm{O}_{4}$ core $\mathrm{Zn}_{x} \mathrm{Co}_{1-x} \mathrm{Fe}_{2} \mathrm{O}_{4}$ shell, the magnetic anisotropy can be systematically decreased as the $\mathrm{Zn}$ concentration increases from $x=0$ to 1.00 . The analysis of the mag- netic properties confirms the rigid coupling of the core and shell magnetic phases that coherently rotate with the external magnetic field. In this way the Néel relaxation time decreases respect to the Brown relaxation and become the dominant heating process at higher $\mathrm{Zn}$ concentration at the shell. We remark that the control of the magnetic anisotropy was done without detriment of the magnetization value, for which it is essential the use of core/shell nanoparticles, where a thin shell determines the magnetic hardening of the material by the interface exchange coupling. The Magnetic Hyperthermia experiments of the nanoparticles diluted in hexane shows that the dominant heating mechanism of the samples with higher anisotropy $(x \leq 0.5)$ is the Brown relaxation, while the predominant mechanism in the samples with lower anisotropy $(x \geq 0.75)$ is the Néel relaxation. These results were confirmed by Magnetic Hyperthermia experiments performed with the nanoparticles dispersed in a media with larger viscosity, i.e. butter oil, where the SPA value is drastically reduced. The obtained data were included in the diagram that considers the relationship between the different relaxation times involved and were interpreted according to the Rosensweig's model, based on the linear response theory. In this way it is possible to understand the evolution of the magnetic hyperthermia as a function of the different involved parameters and predict the optimum condition to obtain the larger specific power absorption. The core/shell morphology proposed in this work, not only allows the fabrication of experimental models to study the relaxation mechanisms and the magnetic anisotropy, but also provides a tool to optimize the SPA, by tuning the proper $\mathrm{Zn}$ concentration, when the Néel relaxation is the dominant mechanism. These results provide a simple and effective way to adjust the magnetic anisotropy to reach the optimal heating condition for a particular environment with a given viscosity, which could be a promising approach for in vivo application of Magnetic Fluid Hyperthermia.

\section{Conflicts of interest}

The authors declare they have no conflicts of interest. 


\section{Acknowledgements}

The authors acknowledge financial support of Argentinian governmental agency ANPCyT (Project No.PICT-2016-0288 and PICT-2015-0883) and UNCuyo (Project No.06/C527 and 06/C528). The authors gratefully acknowledge the EU-commission financial support under the: H2020-MSCA-RISE-2016 SPICOLOST PROJECT No 734187. MRI, GFG and TET also acknowledge the financial support from the Spanish Ministerio de Ciencia, Innovación y Universidades (project MAT2016-78201-P) and the Aragon Regional Government (DGA, Project No. E26).

\section{References}

1 K. Mahmoudi, A. Bouras, D. Bozec, R. Ivkov and C. Hadjipanayis, Int. J. Hyperth., 2018, 0, 1-13.

2 K. Maier-Hauff, F. Ulrich, D. Nestler, H. Niehoff, P. Wust, B. Thiesen, H. Orawa, V. Budach and A. Jordan, J. Neuro-Oncol., 2011, 103, 317-324.

3 J. Shi, P. W. Kantoff, R. Wooster and O. C. Farokhzad, Nat. Rev. Cancer, 2017, 17, 20-37.

4 N. A. Usov and B. Y. Liubimov, J. Appl. Phys., 2012, 112, 023901.

5 R. Rosensweig, J. Magn. Magn. Mater., 2002, 252, 370 -374 .

6 E. Lima, E. De Biasi, R. D. Zysler, M. Vasquez Mansilla, M. L. Mojica-Pisciotti, T. E. Torres, M. P. Calatayud, C. Marquina, M. Ricardo Ibarra and G. F. Goya, J. Nanoparticle Res., 2014, 16, 2791.

7 R. Hergt, S. Dutz and M. Röder, J. Phys. Condens. Matter, 2008, 20, 385214.

8 M. Gonzales-Weimuller, M. Zeisberger and K. M. Krishnan, J. Magn. Magn. Mater., 2009, 321, 1947 - 1950.

9 M. Cruz, L. Ferreira, J. Ramos, S. Mendo, A. Alves, M. Godinho and M. Carvalho, J. Alloy. Comp., 2017, 703, $370-380$.

10 S. Noh, W. Na, J. Jang, J. Lee, E. Lee, S. Moon, Y. Lim, J. Shin and J. Cheon, Nano Lett., 2012, 12, 3716-3721.

11 R. D. Corato, A. Espinosa, L. Lartigue, M. Tharaud, S. Chat, T. Pellegrino, C. Ménager, F. Gazeau and C. Wilhelm, Biomaterials, 2014, 35, 6400 - 6411.

12 S. He, H. Zhang, Y. Liu, F. Sun, X. Yu, X. Li, L. Zhang, L. Wang, K. Mao, G. Wang, Y. Lin, Z. Han, R. Sabirianov and H. Zeng, Small, 2018, 14, 1800135.

13 R. D. Raland, D. Saikia, C. Borgohain and J. P. Borah, J. Phys. D, 2017, 50, 325004.

14 A.-H. Lu, E. Salabas and F. Schüth, Angew. Chem. Int. Ed., 46, 1222-1244.
15 J. Carrey, B. Mehdaoui and M. Respaud, J. Appl. Phys., 2011, 109, 083921.

16 H. Mamiya and B. Jeyadevan, Sci. Rep., 2011, 1, 157.

17 L. Branquinho, M. Carrião, A. Costa, N. Zufelato, M. Sousa, R. Miotto, R. Ivkov and A. Bakuzis, Sci. Rep., 2013, 3, 2887.

18 E. Lima, E. D. Biasi, M. V. Mansilla, M. E. Saleta, M. Granada, H. E. Troiani, F. B. Effenberger, L. M. Rossi, H. R. Rechenberg and R. D. Zysler, J. Phys. D, 2013, 46, 045002.

19 B. Sanz, M. P. Calatayud, E. De Biasi, E. Lima Jr., M. V. Mansilla, R. D. Zysler, M. R. Ibarra and G. F. Goya, Sci. Rep., 2016, 6, 38733.

20 E. A. Périgo, G. Hemery, O. Sandre, D. Ortega, E. Garaio, F. Plazaola and F. J. Teran, Appl. Phys. Rev., 2015, 2, 041302.

21 G. Salas, J. Camarero, D. Cabrera, H. Takacs, M. Varela, R. Ludwig, H. Dähring, I. Hilger, R. Miranda, M. d. P. Morales and F. J. Teran, J. Phys. Chem. C, 2014, 118, 19985-19994.

22 E. Lima Jr., A. L. Brandl, A. D. Arelaro and G. F. Goya, J. Appl. Phys., 2006, 99, 083908.

23 M. Vasilakaki, K. N. Trohidou and J. Nogués, Sci. Rep., 2015, 5, 9609.

24 S. Dutz, M. Kettering, I. Hilger, R. MÃijller and M. Zeisberger, Nanotechnology, 2011, 22, 265102.

25 S. Dutz and R. Hergt, International Journal of Hyperthermia, 2013, 29, 790-800.

26 D. Serantes, K. Simeonidis, M. Angelakeris, O. Chubykalo-Fesenko, M. Marciello, M. d. P. Morales, D. Baldomir and C. Martinez-Boubeta, J. Phys. Chem. C, 2014, 118, 5927-5934.

27 J. Wotschadlo, T. Liebert, T. Heinze, K. Wagner, M. Schnabelrauch, S. Dutz, R. MÃijller, F. Steiniger, M. Schwalbe, T. C. Kroll, K. HÃüffken, N. Buske and J. H. Clement, Journal of Magnetism and Magnetic Materials, 2009, 321, 1469 - 1473.

28 A. López-Ortega, M. Estrader, G. Salazar-Alvarez, A. G. Roca and J. Nogués, Phys. Rep., 2015, 553, 1 - 32.

29 E. Lima, E. L. Winkler, D. Tobia, H. E. Troiani, R. D. Zysler, E. Agostinelli and D. Fiorani, Chem. Mater., 2012, 24, 512-516.

30 G. C. Lavorato, D. Peddis, E. Lima, H. E. Troiani, E. Agostinelli, D. Fiorani, R. D. Zysler and E. L. Winkler, J. Phys. Chem. C, 2015, 119, 15755-15762.

31 R. Skomski and J. M. D. Coey, Phys. Rev. B, 1993, 48, 15812-15816.

32 E. F. Kneller and R. Hawig, IEEE Trans. Magn., 1991, 27, 3588-3560.

33 E. L. Winkler, E. L. Jr., D. Tobia, M. E. Saleta, H. E. 
Troiani, E. Agostinelli, D. Fiorani and R. D. Zysler, Appl. Phys. Lett., 2012, 101, 252405.

34 G. P. Zhao, M. G. Zhao, H. S. Lim, Y. P. Feng and C. K. Ong, Appl. Phys. Lett., 2005, 87, 162513.

35 E. E. Fullerton, J. S. Jiang and S. D. Bader, J. Magn Magn. Mater., 1999, 200, 392 - 404.

36 G. Lavorato, E. Winkler, B. Rivas-Murias and F. Rivadulla, Phys. Rev. B, 2016, 94, 054405.

37 S. I. Mekhtiev, A. A. Mamedov, S. K. Khalilov and M. A. Aleskerov, Izv.Vyssh.Uchebn.Zaved.Neft Gaz, 1975, 64100.

38 H. Hayashi, in Viscoelasticity of Butter, ed. T. Yano, R. Matsuno and K. Nakamura, Springer US, Boston, MA, 1994, pp. 75-77.

39 S. Sun and H. Zeng, J. Am. Chem. Soc., 2002, 124, 8204-8205.

40 G. C. Lavorato, E. Lima, H. E. Troiani, R. D. Zysler and E. L. Winkler, Nanoscale, 2017, 9, 10240-10247.

41 G. C. Lavorato, E. Lima Jr, D. Tobia, D. Fiorani, H. E. Troiani, R. D. Zysler and E. L. W. , Nanotechnology,
2014, 25, 355704.

42 C. Yao, Q. Zeng, G. F. Goya, T. Torres, J. Liu, H. Wu, M. Ge, Y. Zeng, Y. Wang and J. Z. Jiang, J. Phys. Chem. C, 2007, 111, 12274-12278.

43 V. Mameli, A. Musinu, A. Ardu, G. Ennas, D. Peddis, D. Niznansky, C. Sangregorio, C. Innocenti, N. T. K. Thanh and C. Cannas, Nanoscale, 2016, 8, 10124 10137.

44 S. A. Shah, M. Hashmi, S. Alam and A. Shamim, $J$. Magn. Magn. Mater., 2010, 322, 375 - 381.

45 C. E. Rodríguez Torres, G. A. Pasquevich, P. M. Zélis, F. Golmar, S. P. Heluani, S. K. Nayak, W. A. Adeagbo, W. Hergert, M. Hoffmann, A. Ernst, P. Esquinazi and S. J. Stewart, Phys. Rev. B, 2014, 89, 104411.

46 M. L. Mojica Pisciotti, E. Lima Jr., M. Vasquez Mansilla, V. E. Tognoli, H. E. Troiani, A. A. Pasa, T. B. CreczynskiPasa, A. H. Silva, P. Gurman, L. Colombo, G. F. Goya, A. Lamagna and R. D. Zysler, Journal of Biomedical Materials Research Part B: Applied Biomaterials, 2013, 102, 860-868. 